\title{
Overview, Control Strategies, and Lessons Learned in the CDC Response to the 2014-2016 Ebola Epidemic
}

\author{
Beth P. Bell, MD ${ }^{1}$ \\ Inger K. Damon, $\mathrm{MD}, \mathrm{PhD}^{2}$ \\ Daniel B. Jernigan, $\mathrm{MD}^{3}$ \\ Thomas A. Kenyon, $\mathrm{MD}^{4}$ \\ Stuart T. Nichol, $\mathrm{PhD}^{2}$ \\ John P. O'Connor, MS ${ }^{1}$ \\ Jordan W. Tappero, $\mathrm{MD}^{5}$ \\ ${ }^{1}$ Office of the Director, National Center for Emerging and Zoonotic Infectious Diseases, CDC \\ ${ }^{2}$ Division of High-Consequence Pathogens and Pathology, National Center for Emerging and Zoonotic Infectious Diseases, CDC \\ 3 Influenza Division, National Center for Immunization and Respiratory Diseases, CDC \\ ${ }^{4}$ Office of the Director, Center for Global Health, CDC \\ ${ }^{5}$ Division of Global Health Protection, Center for Global Health, CDC
}

Corresponding author: Beth P. Bell, Office of the Director, National Center for Emerging and Zoonotic Infectious Diseases, CDC. Telephone: 404-639-3967; E-mail: BBell@cdc.gov.

\section{Summary}

During 2014-2016, CDC, working with U.S. and international partners, mounted a concerted response to end the unprecedented epidemic of Ebola virus disease (Ebola) in West Africa. CDC's response, which was the largest in the agency's history, was directed simultaneously at controlling the epidemic in West Africa and strengthening preparedness for Ebola in the United States. Although experience in responding to approximately 20 Ebola outbreaks since 1976 had provided CDC and other international responders an understanding of the disease and how to stop its spread, the epidemic in West Africa presented new and formidable challenges. The initial response was slow and complicated for several reasons, including wide geographic spread of cases, poor public health and societal infrastructure, sociodemographic factors, local unfamiliarity with Ebola, and distrust of government and health care workers. In the United States, widespread public alarm erupted after Ebola cases were diagnosed in Dallas, Texas, and New York City, New York. CDC, in collaboration with its U.S. and international counterparts, applied proven public health strategies as well as innovative new approaches to help control the Ebola epidemic in West Africa and strengthen public health readiness in the United States. Lessons learned include the recognition that West African and other countries need effective systems to detect and stop infectious disease threats, the need for stronger international surge capacity for times when countries are overwhelmed by an outbreak, and the importance of improving infection prevention and control in health care settings.

The activities summarized in this report would not have been possible without collaboration with many U.S. and international partners (http://www.cdc.gov/vhflebolaloutbreaks/2014-west-africalpartners.html).

\section{Introduction}

In response to the emergence, rapid spread, and sustained transmission of Ebola virus disease (Ebola) in West Africa during 2014-2016, CDC worked closely with other U.S. government agencies, ministries of health (MoHs), the World Health Organization (WHO), and other international partners as part of an intensive effort to end the epidemic (Figure 1). Multiple factors led to the unprecedented scale of this epidemic, including the wide geographic spread of cases, slow response by the international community, population intermixing and mobility, disease transmission in densely populated urban areas, poor public health and societal infrastructure, local unfamiliarity with the disease, and distrust of government authorities and health care workers (HCWs). As of March 31, 2016, WHO had reported 28,652 suspected, probable, and confirmed Ebola cases, including 11,325 deaths, far exceeding the combined total number of cases reported in approximately 20 previous outbreaks since the 1970s (Table). This report presents an overview of previous Ebola outbreaks and the 2014-2016 epidemic and observations about the epidemic's implications for future public health responses.

\section{Background}

\section{Ebola Outbreaks and Control Strategies, 1976-2014}

Ebola is a rare and often fatal illness caused by viruses of the family Filoviridae, genus Ebolavirus, which has five viruses: Ebola virus (EBOV), Sudan virus, Bundibugyo virus, 
FIGURE 1. CDC's response to the Ebola epidemic, from the first reported cases through the first year after CDC's EOC was activated, and approximate number of reported new cases of Ebola per week — Guinea, Liberia, and Sierra Leone, March 2014-July 2015

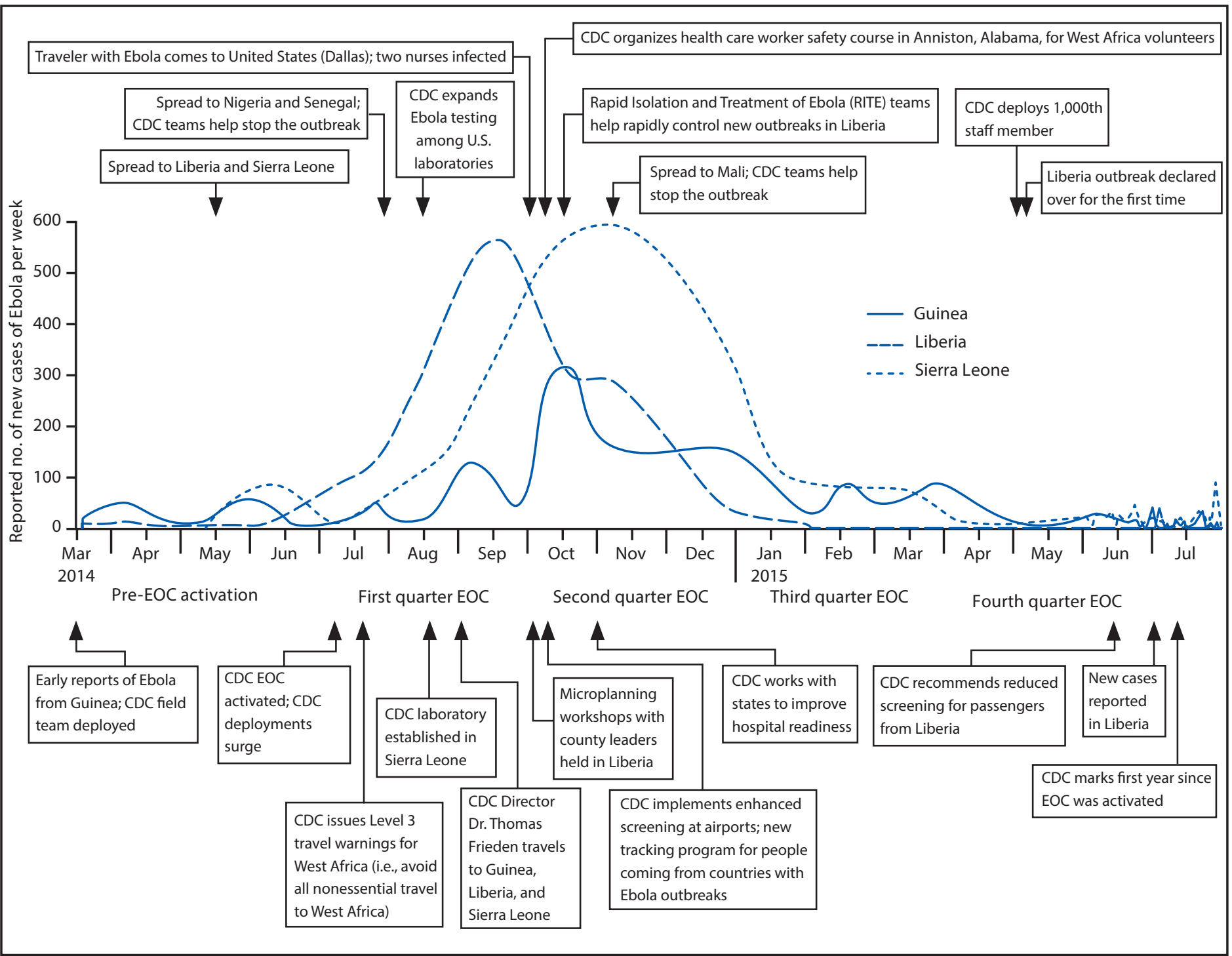

Abbreviations: Ebola = Ebola virus disease; $\mathrm{EOC}=$ Emergency Operations Center.

Taï Forest virus, and Reston virus. All viruses cause disease in humans except Reston virus, which has caused asymptomatic infections in humans but disease in nonhuman primates only. The natural wildlife host of EBOV has not been definitively identified; however, evidence suggests fruit bats of the family Pteropodidae might be a reservoir. Ebola was first recognized in 1976 during two near-simultaneous outbreaks: one caused by EBOV in Zaire (now Democratic Republic of the Congo [DRC]) that comprised 318 cases and 280 deaths (case-fatality rate $[\mathrm{CFR}]=88 \%$ ), and the other caused by Sudan virus in Sudan that comprised 284 cases and 151 deaths (CFR $=53 \%$ ). These and subsequent sporadic outbreaks of Ebola in Eastern and Central African nations (DRC, seven; Uganda, five;
Gabon, four; and Republic of the Congo and Sudan [now South Sudan], three each) had CFRs of approximately 25\%-90\%; occurred in resource-poor settings where health care, transportation, and other services are limited; and lasted from several weeks to approximately 3 months (1) (Table).

EBOV is thought to be introduced into humans when a person has direct contact with blood, body fluids, or organs of infected animals (e.g., fruit bats, chimpanzees, or gorillas) or prepares meat from infected animals. Infection in human communities is sustained through person-to-person contact, often from symptomatic persons to caregivers in homes and health care settings, where infection-control practices are inadequate and personal protective equipment is unavailable 
TABLE. Number of cases and deaths during Ebola outbreaks, excluding the 2014-2016 epidemic — worldwide, 1976-2014

\begin{tabular}{|c|c|c|c|c|c|}
\hline Country & Year & Town & No. of cases & No. of deaths & Species \\
\hline Democratic Republic of the Congo & 2014 & Multiple & 66 & 49 & Zaire ebolavirus \\
\hline Uganda & 2012 & Luwero District & $6^{*}$ & $3^{*}$ & Sudan ebolavirus \\
\hline Democratic Republic of the Congo & 2012 & Isiro Health Zone & $36^{*}$ & $13^{*}$ & Bundibugyo ebolavirus \\
\hline Uganda & 2012 & Kibaale District & $11^{*}$ & $4^{*}$ & Sudan ebolavirus \\
\hline Uganda & 2011 & Luwero District & 1 & 1 & Sudan ebolavirus \\
\hline Democratic Republic of the Congo & 2008 & Luebo & 32 & 15 & Zaire ebolavirus \\
\hline Uganda & 2007 & Bundibugyo & 149 & 37 & Bundibugyo ebolavirus \\
\hline Democratic Republic of the Congo & 2007 & Luebo & 264 & 187 & Zaire ebolavirus \\
\hline South Sudan ${ }^{\dagger}$ & 2004 & Yambio & 17 & 7 & Zaire ebolavirus \\
\hline Republic of the Congo & 2003 & Mbomo & 35 & 29 & Zaire ebolavirus \\
\hline Republic of the Congo & 2002 & Mbomo & 143 & 128 & Zaire ebolavirus \\
\hline Republic of the Congo & 2001 & Not specified & 57 & 43 & Zaire ebolavirus \\
\hline Gabon & 2001 & Libreville & 65 & 53 & Zaire ebolavirus \\
\hline Uganda & 2000 & Gulu & 425 & 224 & Sudan ebolavirus \\
\hline South Africa & 1996 & Johannesburg & 2 & 1 & Zaire ebolavirus \\
\hline Gabon & 1996 & Booue & 60 & 45 & Zaire ebolavirus \\
\hline Gabon & 1996 & Mayibout & 37 & 21 & Zaire ebolavirus \\
\hline Democratic Republic of the Congo ${ }^{\S}$ & 1995 & Kikwit & 315 & 250 & Zaire ebolavirus \\
\hline Côte d'Ivoire & 1994 & Tai Forest & 1 & 0 & Taï Forest ebolavirus \\
\hline Gabon & 1994 & Mekouka & 52 & 31 & Zaire ebolavirus \\
\hline South Sudan ${ }^{\dagger}$ & 1979 & Nzara & 34 & 22 & Sudan ebolavirus \\
\hline Democratic Republic of the Congo & 1977 & Tandala & 1 & 1 & Zaire ebolavirus \\
\hline South Sudan ${ }^{\dagger}$ & 1976 & Nzara & 284 & 151 & Sudan ebolavirus \\
\hline Democratic Republic of the Congo $\S$ & 1976 & Yambuku & 318 & 280 & Zaire ebolavirus \\
\hline
\end{tabular}

Source: CDC. Outbreaks chronology: Ebola virus disease. Atlanta, GA: CDC; 2015. http://www.cdc.gov/vhf/ebola/outbreaks/history/chronology.html

Abbreviation: Ebola $=$ Ebola virus disease.

* Numbers reflect laboratory-confirmed cases only.

${ }^{\dagger}$ Formerly part of Sudan.

$\S$ Formerly Zaire.

or in short supply. In some previous outbreaks (e.g., Kikwit, Zaire, in 1995), the infection cycle was amplified by explosive spread of disease in overcrowded local hospitals, underscoring the role of nosocomial transmission. Because corpses have high viral loads, funerals and burials accompanied by ceremonial washing and touching of deceased persons often are responsible for multiple chains of transmission.

During the first reported Ebola outbreak in Zaire in 1976, an international response team developed an early strategy to stop the outbreak, focusing on the identification, isolation, and care of persons with Ebola symptoms; meticulous contact tracing; engagement with community leaders; culturally sensitive and safe burials; effective infection control; and reliable laboratory testing (2). This strategy, further refined with accumulated experience, has been used to successfully control approximately 20 Ebola outbreaks, including DRC's seventh outbreak in November 2014 (3).

\section{Ebola Symptoms, Tests, Treatment, and Transmission}

Ebola patients typically experience fever, fatigue, muscle pain, and headache, followed by variable signs and symptoms that include vomiting, diarrhea, rash, and hemorrhagic diathesis resulting in external bleeding, internal bleeding, or both. In severe cases, multiorgan dysfunction (e.g., hepatic damage, renal failure, and central nervous system involvement) can develop, leading to shock and death (4). The incubation period is $2-21$ days; symptoms usually appear within $8-10$ days after exposure to EBOV. In the initial clinical phase, Ebola can be difficult to distinguish from other infectious diseases, including malaria, typhoid fever, and Lassa fever.

EBOV infection most commonly is confirmed by testing blood by using a real-time reverse transcription polymerase chain reaction (RT-PCR) assay. Genetic sequencing is increasingly useful for describing the molecular epidemiologic characteristics and other features of Ebola outbreaks. No proven vaccine or specific treatment for Ebola exists; however, human trials of potential vaccines and therapies are under way. Early supportive care with rehydration (e.g., providing intravenous fluids and balancing electrolytes) and treatment of specific symptoms improve chances for survival.

Human-to-human transmission of EBOV occurs through direct contact with the blood or body fluids (e.g., urine, saliva, sweat, feces, vomit, breast milk, or semen) of symptomatic or deceased persons or with objects (e.g., needles and syringes) contaminated with body fluids from an infected person. An infected person becomes contagious once symptoms appear, and the level of infectivity increases dramatically as the disease progresses and the infected person's viral load increases. The 
fluids, skin, and other tissues of persons who die of Ebola are extremely infectious and pose a hazard to anyone who has unprotected contact with the body, including caregivers and people preparing the body for burial. EBOV can be found in the semen of some men who have recovered from the disease, and CDC has recommended that contact with semen from male survivors be avoided until more is known about infectivity of body fluids. If male survivors have sex, they are advised to use a condom correctly and consistently (5).

\section{Emergence in West Africa: A Regional and Global Threat}

The first Ebola cases in West Africa were reported by WHO on March 23, 2014, in the forested rural region of southeastern Guinea bordering Liberia and Sierra Leone, where multiple unrecognized chains of transmission had festered for months (6). The lack of surveillance systems and other public health infrastructure impeded the ability of affected countries to effectively detect and respond to the rapidly evolving outbreak. As the outbreak spread to urban areas and expanded into an epidemic, the number of cases quickly overwhelmed the limited isolation and treatment capacity in the three countries affected, exacerbated by strained laboratory testing capacity. Poor infection control resulted in transmission in health care facilities, including a large number of infections and deaths among HCWs, and collapse of the health care system. Inadequate disease surveillance and reporting further hampered control efforts, resulting in incomplete information about the extent of the outbreak, particularly in difficultto-reach areas. Sociodemographic factors that contributed to virus spread included high mobility and intermixing of populations (e.g., ease of travel across land and river borders) and general unfamiliarity with Ebola and how to respond to Ebola outbreaks. By late July, Ebola had reached the urban and densely populated capitals of all three countries, the first time the disease had caused widespread transmission in crowded metropolitan areas. On August 8, 2014, with case counts steadily increasing, WHO declared the escalating Ebola situation a Public Health Emergency of International Concern (7). By March 2016, WHO had reported cumulative cases throughout Liberia and Sierra Leone and most of the prefectures in Guinea (Figure 2).

\section{CDC's Role and Accomplishments}

CDC's response to the Ebola epidemic was the largest emergency response in the agency's history (8). During CDC's activation of its Emergency Operations Center (EOC) during July 9, 2014-March 31, 2016, approximately 4,000 CDC staff members directly participated in the response, and of these, 1,897 deployed to Guinea, Liberia, Sierra Leone, and other African countries affected by the epidemic (e.g., Nigeria and Mali). CDC's deployed teams included specialists in epidemiology, infection control, laboratory analysis, medical care, emergency management, information technology, health communication, behavioral science, anthropology, logistics, planning, and other disciplines.

\section{Response in Guinea, Liberia, and Sierra Leone}

Before the Ebola epidemic, CDC's presence in Guinea, Liberia, and Sierra Leone was limited to technical support for a small number of disease-specific disease control programs (e.g., malaria and polio) and vaccination campaigns (e.g., measles and yellow fever). In Liberia, CDC also supported the collection of reproductive health data to improve family planning, maternal health, and gender-based violence, as well as programs to help United Nations peacekeepers avoid human immunodeficiency virus infection. In Sierra Leone, CDC evaluated the impact of community case management of childhood diseases programs. Though these programs provided some support for public health systems, they did not include efforts to strengthen infectious disease surveillance with laboratory diagnostic testing. Therefore, CDC's response in these three countries required that CDC experts mobilize from other international and U.S. CDC locations. CDC teams deploying to West Africa early in the response established working relationships with each country's $\mathrm{MoH}, \mathrm{WHO}$, and other international partners. In addition to CDC country teams, CDC staff members in West Africa were part of the Disaster Assistance Response Teams (DARTs) of the U.S. Agency for International Development's Office of Foreign Disaster Assistance, with CDC leading the public health and medical care teams within the DARTs.

A priority was to make response activities faster and more effective. To strengthen coordination among various government and partner organizations in Guinea, Liberia, and Sierra Leone, CDC helped set up national EOCs by using an incident management system (IMS) (9). Teams specializing in areas such as surveillance, case management, infection control, and social mobilization* met daily to report the status of assigned tasks and provide updates on the epidemic to an incident manager, who in turn updated country leaders. Support for EOC buildings, staffing, and operations was provided in large part by the CDC Foundation and by the DARTs and WHO.

\footnotetext{
* Social mobilization is a process that uses dialogue to encourage communities and help them work together to overcome a disease or achieve a social objective.
} 
FIGURE 2. Number of cumulative confirmed Ebola cases during the 2014-2016 Ebola epidemic, by county (Liberia), district (Sierra Leone), and prefecture (Guinea) - Guinea, Liberia, and Sierra Leone, as of March 27, 2016*

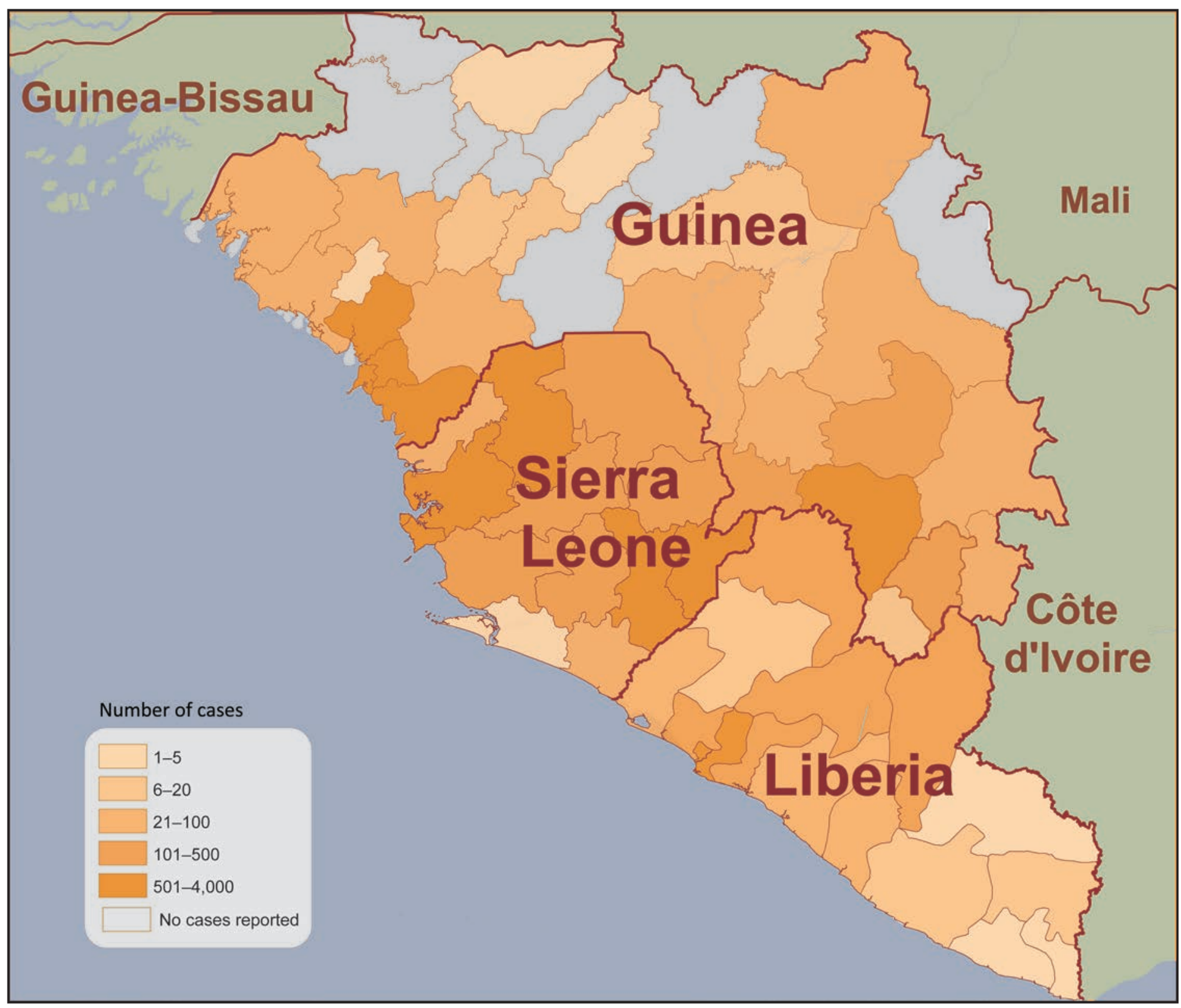

Source: World Health Organization. Ebola situation reports. Geneva, Switzerland:World Health Organization. http://apps.who.int/ebola/ebola-situation-reports Abbreviation: Ebola = Ebola virus disease.

* After March 27, 2016, an additional three confirmed cases were reported in Liberia, and three probable cases were reported in Guinea.

Drawing on experience from previous Ebola responses, CDC worked with governments and partners to detect and break chains of transmission and end the epidemic. Because lack of reliable epidemiologic data was a major challenge from the outset, the teams quickly began to improve surveillance, laboratory, and information management systems to collect, analyze, and report data needed to guide response actions (10). CDC field teams regularly traveled to districts and villages to work with community teams on patient identification and isolation, contact tracing, infection control, social mobilization, and safe burials. However, as the epidemic in West Africa evolved, the large numbers of new cases and contacts each day overwhelmed response efforts. Effective isolation of patients became increasingly difficult as hospitals, clinics, and temporary Ebola treatment units (ETUs) were filled beyond capacity; persons with new suspected cases, as 
well as symptomatic contacts, frequently were turned away from ETUs, thereby fostering new chains of transmission. Suspicion of ETUs as a possible source for infection was common, making some persons reluctant to seek care even when beds were available. In addition, many HCWs became infected and died, contributing to the collapse of an already limited and compromised health care system. The growing numbers of contacts inundated response teams' capacity to identify and monitor contacts. Chains of Ebola transmission evolved rapidly, and responders often were unable to identify how cases were epidemiologically linked.

In September 2014, CDC published results of a modeling analysis that estimated that approximately 555,000 Ebola cases (1.4 million cases when corrected for underreporting) could occur in Liberia and Sierra Leone by January 20, 2015, if approximately $70 \%$ of all persons with new cases were not effectively isolated (11). The model also showed that the speed with which this $70 \%$ target was reached would profoundly affect the total number of cases attributable to the epidemic. As the situation worsened in the three countries that were most heavily affected, these estimates contributed to the decision to massively scale up U.S. resources, including deployment of approximately 3,000 U.S. Department of Defense personnel to Liberia to build ETUs and support other response activities. Other countries and organizations increased their response efforts as well; for example, the African Union mobilized nearly 1,000 African health care staff members to support the response.

CDC teams were integral to each country's EOC in several ways (12). First, they responded rapidly to reports of new cases, helped place symptomatic persons into ETUs, and identified and monitored contacts by creating teams dedicated to targeted and rapid response (e.g., as part of the Rapid Isolation and Treatment of Ebola [RITE] strategy developed in Liberia) (13) and assigning field staff to districts. Second, previous Ebola responses, as well as evidence of widespread transmission in health care settings in the three countries most affected, emphasized the importance of infection control in breaking the chains of EBOV transmission. CDC provided infection-control training to approximately $24,600 \mathrm{HCWs}$ and others; helped establish a system of infection control points of contact in health care facilities in Guinea, Liberia, and Sierra Leone; and conducted 3-day hands-on training in Anniston, Alabama, for approximately 650 U.S. HCWs and other staff scheduled for deployment to West Africa. Third, CDC laboratory experts worked closely with IMS and other response teams to expand and coordinate the availability of laboratory testing of clinical specimens and collaborated to develop faster diagnostic assays. Fourth, social mobilization was used to promote awareness of the epidemic and marshal community religious and political leaders, and CDC health communication experts worked with response teams to help educate local populations about Ebola. Finally, CDC helped each country's MoH develop border and airport exit-screening programs. Teams at national airports screened all passengers before the passengers boarded commercial flights and retained and evaluated travelers with febrile illness to minimize the risk for exportation of Ebola to other countries.

\section{Response in Other African Countries}

In July 2014, a traveler with Ebola flew from Monrovia, Liberia, to Lagos, Nigeria, where multiple responders had unprotected contact with him and were infected, raising the specter of an Ebola epidemic in Africa's most populous city (21 million). The Nigerian government promptly launched an emergency response supported by an existing EOC and IMS structure for polio eradication, Nigerian trainees and graduates of a CDC Field Epidemiology Training Program, and CDC response officials. The IMS response established an ETU within 2 weeks, trained approximately 2,000 Ebola caregivers, identified approximately 890 contacts, and completed 19,000 contact tracing home visits (14). The rapid response helped contain the outbreak to just 19 cases in two cities and averted a public health catastrophe, not only for Nigeria (population approximately 180 million) but also for the entire African continent. WHO declared Nigeria Ebola-free on October 20, 2014.

CDC provided support to Senegal and Mali after separate importations of Ebola into those countries by travelers. Vigorous responses, including meticulous contact tracing, were implemented rapidly, and only a small number of cases occurred (one confirmed in Senegal and eight reported [seven confirmed] in Mali). CDC also worked with $\mathrm{WHO}$ and national $\mathrm{MoH}$ s to improve Ebola preparedness for all at-risk West African countries by helping to plan for EOCs, isolation capacity for patients with suspected Ebola, disease surveillance, laboratory testing, public awareness, and other related activities.

\section{Response in the United States}

As its response in West Africa evolved during the summer of 2014, CDC worked closely with U.S. federal, state, and local public health and clinical partners to prepare for the possible introduction of Ebola into the United States. CDC issued guidance (15) and alerted health care workers to consider a diagnosis of Ebola if patients had compatible symptoms and had visited an affected country within the previous 3 weeks. To facilitate rapid testing, CDC provided staff, training, and support to qualify 56 state and local public health laboratories to perform Ebola reverse transcription polymerase chain reaction (RT-PCR) testing for Ebola. 
The introduction of Ebola into the United States triggered intense national media attention and widespread public alarm. In September 2014, a man flew from Liberia to Dallas, Texas and became ill with Ebola after his arrival; he died in a Dallas hospital. Two nurses who cared for him became infected with Ebola, were hospitalized, and recovered. A fourth U.S. case was confirmed in an HCW who returned from West Africa to New York City in October 2014, was hospitalized there, and recovered; no secondary infections were reported. In addition to these four U.S. patients, seven persons with Ebola symptoms, including six HCWs, were transported by charter aircraft from West Africa to U.S. hospitals; six of these patients recovered.

After the laboratory confirmation of Ebola in the Dallas patient, CDC developed expert teams (i.e., CDC Ebola Response Teams) to deploy where needed anywhere in the United States to assist with the response. To strengthen the preparedness of hospitals nationwide, CDC defined three tiers of hospital readiness, consisting of frontline health care facilities, ${ }^{\dagger}$ Ebola assessment hospitals, and Ebola treatment centers (16). CDC teams with expertise in infection control, occupational health, and laboratory diagnosis visited 81 facilities in 21 states and the District of Columbia (DC) to evaluate their readiness to care for patients with Ebola. By July 2015, a total of 55 hospitals in 17 states and DC were designated by state health departments as Ebola treatment centers.

To improve protection against importation of Ebola into the United States, CDC worked closely with the U.S. Customs and Border Protection (CBP), the U.S. Department of Homeland Security, and state and local public health departments to establish a system to screen and follow up all travelers returning from Ebola-affected countries in West Africa. Travelers arriving from these countries were routed to one of five U.S. airports, triaged by CBP agents, screened for febrile illness, provided with CARE (Check and Report Ebola) kits (consisting of a thermometer, prepaid cell phone, and educational materials), and given an opportunity to have any questions answered by CDC. These returning travelers were then tracked by state and local health departments for any symptoms consistent with Ebola during the 21-day incubation period. During October 2014-December 2015, approximately 29,000 persons were monitored. Health departments also implemented plans to facilitate safe transport of travelers to a hospital ready to assess them for Ebola if fever or other compatible symptoms developed.

\footnotetext{
${ }^{\dagger}$ Facilities able to identify and triage persons under investigation and isolate them, notify the appropriate authorities, and transfer patients to an assessment hospital or treatment center.
}

\section{Conclusion}

The Ebola epidemic of 2014-2016 took a profound toll on the lives of men, women, and children of Guinea, Liberia, and Sierra Leone who were affected by a disease that had never been seen in their part of the world. Although an earlier and more robust response most likely would have controlled the epidemic sooner, the affected West African nations and the international community that responded were not prepared for an epidemic of this magnitude. This epidemic in the three countries and its introduction to seven other countries illustrates how all countries are connected and that a threat in one country is a threat everywhere. Readiness to detect and respond to outbreaks of infectious disease such as Ebola is the goal of the Global Health Security Agenda (GHSA) (17), an initiative supported by countries, government agencies, and international organizations to assist countries with attaining compliance with the International Health Regulations (18) and accelerate progress toward detecting and mitigating infectious disease threats quickly and effectively $(19,20)$. The U.S. government has committed to working in at least 30 countries to implement GHSA, including Guinea, Liberia, and Sierra Leone, where CDC has established new country offices to provide direct technical assistance with implementation. One of the most important lessons of the epidemic is that building these foundational capacities beyond Ebola in the three countries that were most heavily affected and more broadly is pivotal to preventing a similar disaster in the future.

CDC's technical expertise and in-country presence and close collaboration with $\mathrm{MoHs}$ and international partners were vital to controlling the epidemic. By using information gleaned from participation in approximately 20 previous Ebola outbreaks, CDC's Ebola experts and laboratory scientists, emergency management and response specialists, epidemiologists, database developers and managers, health communicators, experts in infection prevention and control and border issues, and numerous dedicated field workers all contributed unique and essential skills. Through rigorous field work to identify and follow up with ill persons and their contacts and innovative and focused epidemiologic analyses, response teams helped demonstrate that the epidemic was more widespread than initially thought and that more extensive and targeted control measures were needed. CDC laboratory scientists staffed field laboratories and helped to boost testing capacity in the three countries that were most heavily affected. CDC worked to strengthen critical control strategies, including case management, meticulous contact tracing, early treatment with supportive care, and social mobilization, and helped to develop 
creative new strategies relevant to this epidemic (e.g., the RITE strategy in Liberia, which enhanced the capacity of county health teams to investigate and lead coordinated responses to outbreaks in remote areas) (13). In-country scientists and public health experts were integral to these activities. CDC's Public Health Ethics Unit staffed the Ethics Desk within the EOC and facilitated consultation on ethical issues.

The epidemic highlighted how much more still needs to be learned about Ebola and the importance of partnerships, including with in-country scientists, in addressing research questions. Some areas of research include less common modes of virus transmission, virus persistence, virus reservoirs, clinical sequelae and disease spectrum, development of faster reliable laboratory tests and genetic analysis methods for virus characterization, improved information technology systems for use in the field, and effectiveness and safety of Ebola therapeutic drugs and vaccines, such as the Sierra Leone Trial to Introduce a Vaccine against Ebola (21).

Guinea, Liberia, and Sierra Leone will move beyond the epidemic and rebuild with resources they lacked before this epidemic, including EOCs, stronger laboratories and surveillance systems, improved infection control in hospitals and health care centers, and better public awareness of the threats posed by infectious diseases. CDC in-country offices have been established and will continue to work with $\mathrm{MoHs}$ and other partners to further strengthen public health systems through GHSA.

CDC's response to the Ebola epidemic in West Africa was made possible by the tireless work, unbridled dedication, and extraordinary resilience of thousands of agency staff members overseas and in the United States. To sustain CDC's readiness to respond to future epidemics, emphasis must continue to be on building capacity and having a strong in-country presence; meticulous field work, technical rigor, and expertise; partnerships with $\mathrm{MoHs}, \mathrm{WHO}$, and other international organizations; and a commitment to effective evidence-based strategies.

\section{Acknowledgment}

CDC Ebola response staff in Atlanta and in affected countries.

\section{References}

1. CDC. Ebola (Ebola virus disease). Atlanta, GA: CDC. http://www.cdc. gov/vhf/ebola/index.html

2. Breman JG, Johnson KM. Ebola then and now. N Engl J Med 2014;371:1663-6. http://dx.doi.org/10.1056/NEJMp1410540
3. World Health Organization. Democratic Republic of Congo: the country that knows how to beat Ebola. Geneva, Switzerland: World Health Organization; 2014. http://www.who.int/features/2014/drc-beats-ebola/en

4. WHO Ebola Response Team. Ebola virus disease in West Africa-the first 9 months of the epidemic and forward projections. N Engl J Med 2014;371:1481-95. http://dx.doi.org/10.1056/NEJMoa1411100

5. CDC. Ebola virus disease transmission. Atlanta, GA: CDC. http://www. cdc.gov/vhf/ebola/transmission

6. Dixon MG, Schafer IJ; CDC. Ebola viral disease outbreak-West Africa, 2014. MMWR Morb Mortal Wkly Rep 2014;63:548-51.

7. World Health Organization. Statement of the 1st meeting of the IHR emergency committee on the 2014 Ebola outbreak in West Africa. Geneva, Switzerland: World Health Organization; 2014. http://www. who.int/mediacentre/news/statements/2014/ebola-20140808/en

8. CDC. The road to zero: CDC's response to the West African Ebola epidemic, 2014-2015. Atlanta, GA: CDC; 2015. http://www.cdc.gov/ about/ebola/index.html

9. Brooks JC, Pinto M, Gill A. Incident management systems and building emergency management capacity during the 2014-2016 Ebola epidemicLiberia, Sierra Leone, and Guinea. In: CDC response to the 2014-2016 Ebola epidemic-_-West Africa and United States. MMWR Suppl 2016;65(No. Suppl 3).

10. Arwady MA, Bawo L, Hunter JC, et al. Evolution of Ebola virus disease from exotic infection to global health priority, Liberia, mid-2014. Emerg Infect Dis 2015;21:578-84. http://dx.doi.org/10.3201/eid2104.141940

11. Meltzer MI, Atkins CY, Santibanez S, et al. Estimating the future number of cases in the Ebola epidemic_-Liberia and Sierra Leone, 2014-2015. MMWR Suppl 2014;63(No. Suppl 3).

12. Frieden TR, Damon IK. Ebola in West Africa-CDC's role in epidemic detection, control, and prevention. Emerg Infect Dis 2015;21:1897-905. http://dx.doi.org/10.3201/eid2111.150949

13. Kateh F, Nagbe T, Kieta A, et al. Rapid response to Ebola outbreaks in remote areas-Liberia, July-November 2014. MMWR Morb Mortal Wkly Rep 2015;64:188-92.

14. Shuaib F, Gunnala R, Musa EO, et al. Ebola virus disease outbreakNigeria, July-September 2014. MMWR Morb Mortal Wkly Rep 2014;63:867-72.

15. CDC. Guidelines for evaluation of U.S. patients suspected of having Ebola virus disease. CDC Health Advisory. Atlanta, GA: CDC; 2014. http://emergency.cdc.gov/han/han00364.asp

16. Van Beneden CA, Pietz H, Kirkcaldy RD, et al. Early identification and prevention of the spread of Ebola-United States. In: CDC response to the 2014-2016 Ebola epidemic-West Africa and United States. MMWR Suppl 2016;65(No. Suppl 3).

17. CDC. The global health security agenda. Atlanta, GA: CDC. http:// www.hhs.gov/about/agencies/oga

18. World Health Organization. International health regulations. Geneva, Switzerland: World Health Organization. http://www.who.int/topics/ international_health_regulations/en

19. Heymann DL, Chen L, Takemi K, et al. Global health security: the wider lessons from the West African Ebola virus disease epidemic. Lancet 2015;385:1884-901. http://dx.doi.org/10.1016/S0140-6736(15)60858-3

20. Frieden TR, Tappero JW, Dowell SF, Hien NT, Guillaume FD, Aceng JR. Safer countries through global health security. Lancet 2014;383:7646. http://dx.doi.org/10.1016/S0140-6736(14)60189-6

21. Widdowson MA, Schrag SJ, Carter RJ, et al. Implementing an Ebola vaccine study-Sierra Leone. In: CDC response to the 2014-2016 Ebola epidemicWest Africa and United States. MMWR Suppl 2016;65(No. Suppl 3). 